\title{
Overcoming Cultural Barriers in Undergraduate Nursing Education Using Voice Enhanced High Fidelity Simulation: The Sultan Qaboos University Experience
}

\author{
Gerald Amandu Matua, Divya Raghavan, Vidya Seshan, Arwa Atef Sultan Obeidat \\ Sultan Qaboos University, Oman
}

\begin{abstract}
Cultural barriers can significantly diminish educator's chances of teaching clinical skills and competencies to students [1]. We report about Voice Enhanced High Fidelity Simulation (VES) using Gaumard's NOELLE® Advanced Maternal Care simulator to teach undergraduate male nursing students maternity nursing skills. This innovation was essential because as a minimum entry-topractice competency, baccalaureate nursing graduates are required to competently care for mothers and their families during labor and childbirth, and to provide safe and supportive environment, while being able to identify complications and respond to psychological needs [2][3]. In our study, we found that simulation helped students to fill the gap between theoretical knowledge and practical skills. Secondly, simulation helped the students to experience heightened awareness and deeper appreciation of the process of labour and childbirth. Thirdly, simulation enhanced student's communication ability with the mother. Furthermore, simulated experiences taught the students when to call for help. Finally, simulation enabled the students to better understand the role and tenets of interprofessional collaboration in the management of labour. We conclude that VES can be used to overcome barriers that hinder the teaching of male nursing student's attitudes, skills and competencies to provide safe care to childbearing mothers and their families, including the tenets of how to effectively collaborate with others during their care.
\end{abstract}

\section{Introduction}

Cultural barriers can significantly diminish educator's chances of teaching practical skills and competencies to their students [1]. In healthcare practice disciplines like nursing and medicine, such barriers often hinder student's learning of essential clinical skills. We highlight the innovative approach we adopted to overcome the cultural barriers that had hindered the effective teaching of our male nursing students' caring and interprofessional practice behaviours and competencies in maternity nursing course which is a mandatory course in our undergraduate nursing program.

A remedy to this cultural barrier, we adopted the use of Gaumard's NOELLE® Advanced Maternal Care simulator to provide our male nursing students near-real-life clinical experience to teach the students the essential skills and competencies for maternity nursing. This innovation was essential because as a minimum entry-to-practice competency, graduates of baccalaureate nursing programs are required to competently care for pregnant mothers and their families during labour and childbirth in a safe and supportive care environment; while being able to competently identify any unforeseen complications and promptly respond to their psychological needs as well [2][3].

In order to acquire the necessary competencies, undergraduate nursing students are usually required to obtain various psychomotor abilities, in which repeated practice is required to accomplish the essential expertise over the span of their initial training. However, in some disciplines like maternity nursing, transmitting the necessary competencies and the accompanying psychomotor abilities is hindered by cultural factors. This cultural barrier disproportionately targets male nursing students especially in relation to skills that need to be acquired from clinical areas such as labour and postnatal wards that are considered to be sensitive because of the intimate nature of the care required while caring for pregnant mothers during labour and childbirth [1][4].

As a result of this cultural barrier, male nursing and medical students tend to have significantly less clinical experience than female students in key areas of obstetrics and gynecology where they experience discrimination from clinical instructors or clinicians or even patients themselves. In essence, male healthcare students tend to experience a higher rate of refusals by patients when compared to female students. In fact, a recent study conducted in Malaysia confirms this trend. The investigation [5] involving attendees of gynecology clinic, found that male medical students experienced rejection $87 \%$ more compared to $32 \%$ by female students, when the patients were asked who they would allow to observe their genital area and perform a pelvic examination.

This finding therefore suggests that in order to improve the clinical learning experience of male nursing and medical students, the gender discrimination and educational inequities experienced by the male students should be significantly minimized or alternatives should be designed [1][5]. This recommendation is particularly vital because in Oman, like in most Middle Eastern 
cultures, non-related males are not allowed to have physical contact with females [6].

This cultural practice prevents male students from being placed in hospitals where they can witness and care for child bearing mothers during labour, delivery and in the postnatal period. The irony is that while this trend has continued to prevail, to the contrary all undergraduate nursing and medical students, regardless of their gender, are expected to poses the minimum entry-to-practice competencies required to safely care for all patients including pregnant mothers during labour and childbirth [7].

In effort to address this shortcoming and enhance skills transfer to their students, many educators are increasingly resorting to using high fidelity simulated experience as a strategy to increase their students' skill and competences. Simulation is an approach to teaching and learning which presents to the learner through a simulated patient the opportunity to interact with the patient, colleagues and others to gain desired actions and skills intended by the educator. In simulation, the trainer tactfully recreates characteristics of the real world in a carefully controlled learning environment while optimizing the conditions for the skill being taught to be best assimilated by the learner [8].

Consistently, the outcome of properly implemented simulated learning experiences have been found to be effective and rewarding for both the trainer and the trainee [9]. It therefore comes as no surprise that this opportunity simulated learning creates for the trainer to afford students to learn in a safe and well controlled environment without harming patients in the process of learning critical skills has led the General Medical Council of United Kingdom to recommend that medical schools should use simulation technology in the education of undergraduate medical students [10].

As a result of these positive outcomes, simulation, using high fidelity technologies such as NOELLE® Advanced Maternal Care simulator is becoming a prominent strategy throughout the healthcare professionals education world especially among nursing and medical educators because of its ability to closely replicate desired clinical experiences in a safe and authentic manner, thereby resulting in near real-life learning resulting in increased skills acquisition in the learners [8] [11].

This drive towards more integration of simulation in healthcare curriculum is the result of the realization by educators that creating meaningful and robust learning experiences through simulation can benefit students' performance in subsequent clinical practice. This observation is particularly important because simulation provides the opportunity to rehearse particular clinical scenarios, which may be otherwise difficult to achieve. It also allows students to anticipate likely patient prognosis and appreciate how best to respond to patients, relatives, and significant others within the healthcare arena when they become exposed to the actual clinical situation [8] [9] [12].

\section{Problem Statement}

While simulation-based teaching has gained momentum in nursing education, however some researchers have continued to question its effectiveness in terms of transfer of knowledge and skills to the students. There is thus a need for valid and reliable research to quantify the educational effect of simulation, especially in circumstances where there is cultural or social barrier for male nursing students to practice maternity nursing in the clinical setting.

In our context, maternity nursing clinical rotation has been a challenge for male nursing student since most Middle Eastern cultures do not allow them to assist mothers either as a nurse or midwife during childbirth [6] [13].

\section{Study Aim}

To examine if Voice enhanced high fidelity simulation using NOELLE® Advanced Maternal Care simulator enhances academic performance of male undergraduate nursing students.

\section{Specific Objectives}

To explore the male nursing student's perception of their clinical competence to effectively manage women in labor through voice enhanced high fidelity simulation simulated experiences.

\section{Research Question}

How do male nursing students perceive their level of competence to manage pregnant women in labor achieved through voice enhanced high fidelity simulated learning experiences?

\section{Research Design}

We used a generic qualitative design using the focus group discussion method because there is no much of information and data available related to this topic in the Middle East at the time of conducting this study. Like in this study, focus group discussion method is frequently used as a research approach to gain an in-depth understanding of poorly understood phenomena.

In this method, data is generated from a purposely selected group of individuals rather than from a statistically representative sample of a 
broader population. This method allowed the participants to express their experience in own words. Furthermore, the approach also allowed the researchers to integrate the participant's reported experiences with the researchers' own observations [14].

\section{Eligibility Criteria}

To be eligible for our study the participants had to be:

a) registered in the undergraduate nursing program at the College of Nursing.

b) registered for Maternity Nursing Course in the years 2017 and 2018.

c) willing to participate in the study voluntarily and ready to share views freely.

d) willing to sign the informed consent form as evidence of voluntary participation.

\section{Population}

The study population included all the Male Nursing Students in the Baccalaureate Nursing Program enrolled for Maternity Nursing Clinical Course in the academic years 2017 and 2018.

\section{Sample}

The study sample included all the male nursing students who agreed to participate in the study by signing a written informed consent. In total, twentythree (23) students participated in the study, through five focus groups, with each focus group comprising between 4-6 students per group.

\section{Setting}

The study took place at College of Nursing; Sultan Qaboos University, Sultanate of Oman's premier public university. The interviews occurred in the teaching rooms after official class hours.

\section{Ethical Considerations}

Permission to conduct the study was granted by the College Research and Ethics Committee (IRB\# Protocol 14/2017). All participants voluntarily agreed to participate and signed informed consent forms before the study. Participant' anonymity and confidentiality of their data was strictly upheld throughout the interviews and during data analysis phase of the study. To enhance confidentiality and anonymity, each participant was given a code number, which was used for data analysis and reporting. The focus group interviews were conducted by the research team member well experienced in conducting focus group interviews and was not part of the maternity teaching team.

\section{Research Intervention}

After teaching the theoretical aspects of the labor process, the one of the researchers, a clinical instructor in the course, demonstrated to the students practically, the process of labor using Gaumard's NOELLE® Advanced Maternal Care simulator.

To enhance skill retention, the following day, each participant had a follow up hands-on experience of labor using NOELLE® high-fidelity simulator. In the practical sessions, the patient environment was arranged to closely resemble the reality of the labor room in a standard hospital.

In addition to recreating the labor room environment, one of the researchers, added emotion to the birthing process, by mimicking the sounds mothers in labor would usually make that correspond to the different stages of labor and child birth including when they experienced complications.

The instructors recreated a near-real-labor room environment both physically and psychologically. Hence, as the students assisted with delivery, they were subjected to different stages of labor including complications, with the instructor "voicing over" the different situations experienced.

\section{Data Collection}

Data was collected during the academic years 2017 and 2018, after the target population was taught the theory of labor process and the simulated management of labor and birth process as part of their maternity nursing clinical course.

In line with ethical requirement, each participant was informed about the purpose, benefits and the risks involved and those who agreed to participate in the study signed an informed consent form as evidence that they participation was voluntary.

Each of the five-focus group consisted of between 4-6 students, with each interview lasting between 60 to 90 minutes. As a best practice, all the interviews were audio recorded in addition to extensive field notes taken by one of the researchers.

In order to ensure that the interviews did not negatively affect the students, the focus group interviews were scheduled according to participant's convenience outside class hours. The group discussions continued until data saturation occurred.

\section{Data Analysis}

Data analysis commenced after the verbatim transcription of the focus group discussions interviews were integrated with the extensive field notes into the final data set of the investigation. 
Data was analyzed thematically, and the process involved each research team member individually reading the transcripts several times and then identifying thematic statements. The various thematic statements were later compared, discussed and agreed upon among the four researchers.

\section{Findings}

Participants perceived their level of competence to manage pregnant women during the different phases of labor and childbirth through five "thematic areas" as discussed subsequently:

\subsection{Theme \# 1- Bridging the Gap between Theory and Practice}

The simulation sessions helped participants to "bridging the gap between theoretical knowledge and practical skills" (P3), adding that as they conducted the simulated deliveries, the "live" voice over performed by the instructor made them feel as if they were assisting real mothers.

In further support of the VES strategy, another participant declared that:

...the distress calls gave us cues on what to do to address each mother's needs (P1).

\subsection{Theme\# 2: Better appreciation of labour and child bearing experience}

Participants experienced heightened awareness and deeper appreciation of the labor and child bearing experience, than they did previously. They attributed their improved clinical performance and higher confidence levels to the two simulated learning sessions, adding that the "voice" cues helped them to better appreciate the different stages of labor including the care at every stage.

In support of this finding, a participant declared:

"...the scenario felt so real and the patient was so much in pain. I wanted the scenario to end as soon as possible. I had to help her because the pain was so much and I wanted it to end soon" (P.14).

\subsection{Theme\# 3: Enhanced communication ability during labour and childbirth}

Participants experienced high level of competence as evidenced by their enhanced communication ability as they care for the mother. The participants declared that the "live" "distress calls" which signified conditions such as pain, bleeding and complications taught them how to communicate more professionally to the mother, her family members and other members of the healthcare team.

In support, a participant described that:

"the voice helped me to provide accurate information about the stage of labour or complication the mother was experiencing to her family and other caregivers (P.12).

\subsection{Theme \#4: Understanding when to call for help}

Participants reported that they appreciated "when to call for help" when assisting mothers during labor and childbirth. They asserted that with voice cues, they knew exactly what to do, especially when complications occurred. This was a vital revelation since complications during labor or childbirth are often worsened by primary caregivers delaying to seek lifesaving help from others.

In support, a student described that:

"The simulations taught me when I need to ask for others' help”. (P.16)

A second participant added that:

“'Once you see complications...it's clear you need to seek for help right away (P.12).

\subsection{Theme\# 5: Understanding the role of interdisciplinary collaboration}

Participants reported that the simulation sessions helped them to better understand the role and tenets of interdisciplinary care and interprofessional collaboration, as this student recounted:

“...when she developed complications, I called the specialist to manage her ... (P.11).

In like manner, another participant declared that:

“...when complications occur, it's clear you need a specialists' intervention ...(P.5).

\section{Discussion}

We found that VES increased participant's level of competence to manage pregnant women in labor. This observation is supported by all the five thematic areas, ranging from the simulated learning experiences 'bridging the gap between their 
theoretical knowledge and practical skills' (theme 1) to 'understanding the role of interdisciplinary collaboration' (theme 5). This meant they acquired the essential skills and competencies to care for mothers in labor and childbirth.

In line with theme one, the participants felt that simulation helped them to "bridge the gap between their theoretical knowledge and practical skills". It was clear from their recollections that as they conducted the simulated deliveries, the "live" voice over performed by instructor made them feel as if they were assisting real mothers. This innovative approach of recreating reality in the learning of essential maternity skills appears to enable the students to better respond to mothers' needs.

Parsons, et al [15] in their study involving Simulation-based Crisis Resource Management Training for Emergency Medicine Residents reported similar findings. In their study, the researchers observed that the medical residents after their simulated training course displayed improved skills in leadership, problem solving, communication, situational awareness, teamwork, resource utilization. This finding further points to simulation as tool for enabling skill acquisition.

In regard to theme two, the participants demonstrated "better appreciation of labour and child bearing experience". This better appreciation seems to have translated into better performance of their care due to the increased confidence levels following the simulation sessions. This claim of enhanced performance was verified by instructors and reinforced by comparable course grades of male and female students. This theme further exemplifies the transformation simulation can bring.

This finding agrees with that of Zapko and others [16] who report that properly conducted serial simulated learning experiences are a strong learning tool. The researchers in this USA based research university found that that the students involved in their study were satisfied with simulation experience, were confident in their skills performance, and felt that simulation was based on sound educational practices and enhanced their learning. The researchers concluded that if properly conducted, simulations can lead to increased student satisfaction and self-confidence.

The third theme relates to participant's "enhanced communication ability during labor and childbirth". These findings is a welcome outcome since communication during labor and delivery is a vital aspect of care to the birthing mother. The participants attributed this realization to the "live" distress calls of NOELLE® which matched conditions like pain, bleeding or other complications she was experiencing. Participants suggest that the "distress call", heightened their awareness to communicate more effectively to the mother, her family and other members of the healthcare team.
Foronda and colleagues [17] report consistent findings when the researchers subjected baccalaureate nursing students to the use of virtual clinical simulation to improve communication skills. In this USA based study, the researchers report through field notes that they observed students listening to how their peers communicated and learned from them. The investigators reported that the participants reported less anxiety, knew better what to expect, and had "better flow" with communication to peers and to the physician following their simulated experiences.

In another USA based project, this time involving intensive care unit nurses, the researchers using a multifaceted educational strategy with high-fidelity simulation to introduce evidence-based communication tools to the nurses report that the nurses were satisfied with the education strategy, and their perceptions of interprofessional communication and knowledge improved subsequently. Studies such as these further underscores the capacity of simulation as a reliable tool for teaching communication skills to both undergraduate and practicing healthcare professionals [18].

The fourth finding reflected in theme four, highlights the male students' "understanding when to call for help" when confronted with challenges while assisting mothers in labor and childbirth. This was a vital revelation since complications often develop during this period and primary caregivers must quickly detect when to ask for support from others within the healthcare team.

Interestingly, a study conducted in the Institute of Clinical Science, Queen's University Belfast, Northern Ireland, among nursing and medical students reported comparable results. In this study, both the medical and nursing students felt that the high fidelity pediatric simulation they has been subjected to improved their clinical and practice-based skills, and helped them to appreciate the role of each other. Remarkably, both groups of students called for more interprofessional and simulation learning opportunities, indicating a positive attitude towards simulated learning [19].

The fifth finding corresponding to theme five, highlights that the simulation sessions helped students to better understand "the role of interdisciplinary collaboration" in managing mothers in labor and childbirth. The participants' recounted that the simulated management of various complications enabled them to better appreciate the role each healthcare team member played in managing such complications.

This finding agrees with what Watters and team [20] report in their study published in the British Medical Journal Open involving nurses and physicians. The researchers revealed that highfidelity simulation training increased nurses and physicians' perceived abilities related to teamwork 
and leadership and management of clinical scenarios. This conclusion was based on the researcher's observation that thematic analysis of qualitative data showed statistical improvements in communication and teamwork and leadership for both doctors and nurses undergoing simulation training, thereby further underscoring the value of simulation.

\section{Limitations}

These findings are limited to the application of the Voice Enhanced Simulation (VES) to teaching of maternity nursing skills to undergraduate nursing students; hence the findings must be used with caution when applied to other areas outside nursing or midwifery for undergraduate skills training.

\section{Conclusions}

The researchers conclude that when properly implemented, VES:

- Helps educators to overcome barriers hindering the teaching of male nursing students, attitudes, skills and competencies to provide safe care to mothers during labor and childbirth.

- Provides a credible avenue through which students can learn to bridge the gap between theoretical knowledge and the actual practical skills expected of them in the clinical area.

- Enables novice students to appreciate the nuances of labor and childbirth in order to appropriately respond physically and psychologically to the needs of the childbearing family.

- Enhance the communication ability of undergraduate nursing students involved in the care of childbearing family thereby making them safer and more effective healthcare practitioners.

- Helps undergraduate nursing students to understand their limit of competence and know when they should seek for help when confronted with complications during labor and childbirth.

- Provides a safe environment where undergraduate nursing students can learn the role of other healthcare professionals in order collaborate more effectively with them in caring for mothers.

\section{Recommendations}

On the basis of the findings, the researchers recommend the following:
- Since VES helps to create a learning environment that results in improved student performance and clinical skills, educators in undergraduate nursing and related educational programs should consider adopting the strategy to teach essential clinical skills to their undergraduate students.

- Additional research using VES is recommended in other clinical courses apart from maternity nursing to help generalize these findings to other nursing and healthcare related courses.

- We further recommend a quantitative study to examine in more specific ways how other aspects of the VES strategy are linked to student performance as a more concrete step to increase its generalizability and application across caring disciplines for wider adoption.

\section{Acknowledgement}

We are grateful to the College of Nursing, Sultan Qaboos University for funding this research study and for supporting the corresponding author to attend the 2019 Canada International Conference on Education from June 22- 25, 2019 in University of Toronto in Mississauga, Canada.

\section{Reference}

[1] Keogh, B., \& O'lynn, C. (2007). Male nurses' experiences of gender barriers: Irish and American perspectives. Nurse Educator, 32(6), 256-259.

[2] MacKinnon, K. (2011). Rural nurses' safeguarding work: Re-embodying patient safety. Advances in Nursing Science, 34(2), 119-129.

[3] Adejumo, O., \& Mukumbang, F. C. (2014). Patients' experiences of being nursed by student nurses at a teaching hospital. Curationis 37(1), 1230, 10.

[4] Golden, S. E. (2018). Strategies to Overcome Gender Bias in Maternity Nursing. Nursing for women's health, 22(5), 366-371.

[5] Zahid, A. Z. M., Ismail, Z., Abdullah, B., \& Daud, S. (2015). Gender bias in training of medical students in obstetrics and gynaecology: a myth or reality?. European Journal of Obstetrics \& Gynecology and Reproductive Biology, 186, 17-21.

[6] Al Omoush, K. S., Yaseen, S. G., \& Alma'Aitah, M. A. (2012). The impact of Arab cultural values on online social networking: The case of Facebook. Computers in Human Behavior, 28(6), 2387-2399. 
[7] Satu, K. U., Leena, S., Mikko, S., Riitta, S., \& Helena, L. K. (2013). Competence areas of nursing students in Europe. Nurse education today, 33(6), 625-632.

[8] Beaubien, J. M., \& Baker, D. P. (2004). The use of simulation for training teamwork skills in health care: how low can you go? BMJ Quality \& Safety, 13(suppl 1), i51i56.

[9] Beal, M. D., Kinnear, J., Anderson, C. R., Martin, T. D., Wamboldt, R., \& Hooper, L. (2017). The effectiveness of medical simulation in teaching medical students critical care medicine: a systematic review and meta-analysis. Simulation in Healthcare, 12(2), 104-116.

[10] General Medical Council 2009. Tomorrow's Doctors. London: General Medical Council. Retrieved [15 July 2019] from http://www.ub.edu/medicina_ unitateducaciomedica/documentos/TomorrowsDoctors_20 09.pdf

[11] Curl, E. D., Smith, S., Chisholm, L. A., McGee, L. A., \& Das, K. (2016). Effectiveness of integrated simulation and clinical experiences compared to traditional clinical experiences for nursing students. Nursing Education Perspectives, 37(2), 72-77.

[12] Adams, J., Cepeda Brito, J. R., Baker, L., Hughes, P. G., Gothard, M. D., McCarroll, M. L., Davis, J, Silber, A, \& Ahmed, R. A. (2016). Management of maternal cardiac arrest in the third trimester of pregnancy: a simulationbased pilot study. Critical care research and practice, 2016.

[13] Herrmann-Werner, A., Nikendei, C., Keifenheim, K., Bosse, H. M., Lund, F., Wagner, R., ... \& Weyrich, P. (2013). "Best practice" skills lab training vs. a "see one, do one" approach in undergraduate medical education: an RCT on students' long-term ability to perform procedural clinical skills. PloS one, 8(9), e76354.

[14] Nyumba, T., Wilson, K., Derrick, C. J., \& Mukherjee, N. (2018). The use of focus group discussion methodology: Insights from two decades of application in conservation. Methods in Ecology and evolution, 9(1), 2032.

[15] Parsons, J. R., Crichlow, A., Ponnuru, S., Shewokis, P. A., Goswami, V., \& Griswold, S. (2018). Filling the gap: simulation-based crisis resource management training for emergency medicine residents. Western Journal of Emergency Medicine, 19(1), 205.

[16] Zapko, K. A., Ferranto, M. L. G., Blasiman, R., \& Shelestak, D. (2018). Evaluating best educational practices, student satisfaction, and self-confidence in simulation: A descriptive study. Nurse education today, 60 , 28-34.

[17] Foronda, C., Gattamorta, K., Snowden, K., \& Bauman, E. B. (2014). Use of virtual clinical simulation to improve communication skills of baccalaureate nursing students: A pilot study. Nurse education today, 34(6), e53e57.

[18] Turkelson, C., Aebersold, M., Redman, R., \& Tschannen, D. (2017). Improving nursing communication skills in an intensive care unit using simulation and nursing crew resource management strategies. Journal of nursing care quality, 32(4), 331-339.

[19] Stewart, M., Kennedy, N., \& Cuene-Grandidier, H. (2010). Undergraduate interprofessional education using high-fidelity paediatric simulation. The Clinical Teacher, 7(2), 90-96.

[20] Watters, C., Reedy, G., Ross, A., Morgan, N. J., Handslip, R., \& Jaye, P. (2015). Does interprofessional simulation increase self-efficacy: a comparative study. BMJ open, 5(1), e005472. 